\title{
e-Phaïstos
}

e-Phaïstos Revue d'histoire des techniques / Journal of the history of technology

II-2 | 2013

Les sources de l'histoire des techniques (2)

\section{Les aqueducs à souterazi de la Régence d'Alger}

The Souterazi Aqueducts in the Regency of Algiers

\section{Dalila Kameche-Ouzidane}

\section{OpenEdition}

\section{Journals}

Édition électronique

URL : http://journals.openedition.org/ephaistos/7388

DOI : 10.4000/ephaistos.7388

ISSN : 2552-0741

\section{Éditeur}

IHMC - Institut d'histoire moderne et contemporaine (UMR 8066)

\section{Édition imprimée}

Date de publication : 15 décembre 2013

Pagination : 73-84

ISSN : 2262-7340

\section{Référence électronique}

Dalila Kameche-Ouzidane, "Les aqueducs à souterazi de la Régence d'Alger », e-Phaïstos [En ligne],

II-2 | 2013, mis en ligne le 12 décembre 2019, consulté le 10 décembre 2020. URL : http://

journals.openedition.org/ephaistos/7388; DOI : https://doi.org/10.4000/ephaistos.7388 


\title{
Les aqueducs à souterazi de la Régence d'Alger
}

\author{
Dalila Kameche-Ouzidane \\ Docteur en Histoire des Techniques du Conserva- \\ toire National des Arts et Métiers de Paris. CDHTE- \\ Cnam \\ Laboratoire : Histoire, technique, technologie, pa- \\ trimoine \\ Architecte et enseignante-chercheure à I'EPAU \\ (Ecole Polytechnique d'Architecture et d'Urbanisme \\ d'Alger) /CDHTE-Cnam
}

Sous la Régence (1516-1832), la ville d'Alger comme de nombreuses villes ottomanes est alimentée en eau par un ensemble d'aqueducs. Sa singularité est précisément de devoir ces infrastructures aux gouvernants turcs, contrairement à Alep et Tunis, deux autres grandes villes de l'empire qui les ont héritées de périodes antérieures ${ }^{1}$. Dans une première phase, les Turcs réalisent, entre le milieu du XVIe siècle jusqu'à la première moitié du XVIIe, quatre aqueducs principaux pour alimenter en eau la médina. Dans une deuxième phase, trois aqueducs suburbains sont édifiés pour alimenter en eau le $F a ̂ h s^{2}$ le plus peuplé de la campagne algéroise, soit les localités de Birkhadem et de Birmandreis3, puis la zone côtière de la Pointe Pescade (Mers Eddeben). En 1833, le capitaine Claude-Antoine Rozet4, géologue attaché à l'armée française en qualité d'ingénieur géographe, évalue à plus de deux cents l'ensemble des aqueducs de la ville et des campagnes.

Les aqueducs de la Régence d'Alger sont forts peu connus, à la différence des foggaras ou qanâts du Sud algérien qui exercent une fascination sur les ingénieurs et hydrauliciens du XIX ${ }^{\mathrm{e}}$ siècle.

\section{Origine des eaux d'Alger}

Les eaux qui alimentent l'ancienne médina d'Alger se composent essentiellement des eaux du Sahel 5 , des sources du Télemly, près du Palais d'Été de Mustapha Pacha ${ }^{6}$, ainsi que celles de la nappe du Hamma7, du Café d'Hydra, de Ben Aknoun, de Birtraria. Au début du XXe siècle, Émile Ficheur, professeur de géologie à l'École des sciences d'Alger, auteur de la première carte géologique de la capitale datée de $1904^{8}$, donne un aperçu de l'hydrogéologie du massif ancien sur lequel a été fondée El Djezaïr. Il précise que les sources qui y sont disséminées sont alimentées par les affleurements calcaires, les zones de gneiss et les filons de granulite au contact des assises schisteuses. En outre, les éboulis des pentes et les dépôts d'entraînement du fond des ravins renferment de petites nappes qui alimentent les puits dans une partie du massif schisteux 9 . L'exploitation de l'aquifère se fait aussi grâce aux puits domestiques et quelques puits publics. Globalement, on peut affirmer que chaque maison dispose d'un puits creusé dans le gneiss ou le schiste ; certains ont une profondeur de $50 \mathrm{~m}$, pouvant 
même atteindre $70 \mathrm{~m}$ entre la «Citadelle » et le pied de la colline que constitue la médina. L'hiver, la hauteur d'eau dans ces puits s'élève jusqu'à $30 \mathrm{~m}$, tandis qu'en été, le niveau est si faible qu'il est difficile de tirer l'eau ${ }^{10}$. Cette nappe est également exploitée à l'aide de machines élévatoires, généralement des norias à manège à traction animale. Ces machines versent l'eau dans des bassins d'où partent des canalisations pour irriguer ou gonfler le débit des aqueducs. Par ailleurs, d'ingénieux travaux de galeries filtrantes collectent les moindres filets aquifères. Ludovic Ville explique ce mode de collecte des eaux de sources qui grossissent le flux des aqueducs :

«Â Alger les Maures se sont procuré de nouvelles sources et ont augmenté le débit des sources existantes, en exécutant des galeries à la séparation d'une couche d'argile imperméable et des terrains qui la recouvraient ${ }^{11}$.

L'eau est ensuite acheminée au cour de la cité, puis stockée intramuros auprès des portes comme dans le centre. Des réservoirs partent de nouvelles canalisations qui courent sous les rues, alimentant les fontaines.

\section{Les eaux dans la médina}

Les sources, les puits et les citernes

Intramuros, la distribution d'eau se développe sous la forme d'un système arborescent : les aqueducs courent le long des rues principales puis se subdivisent en branches qui distribuent l'eau à des conduites secondaires et tertiaires à près de 150 fontaines publiques ${ }^{12}$ notamment les fontaines d'ablutions des mosquées, celles des casernes et des palais. On dénombre neuf sources autour et dans la ville $^{13}$. Certaines jouissent d'une réputation particulière telle la Fontaine du Dey (Aïn as-Sûltan), au quartier Fontaine-Fraîche, à l'extérieur de la porte de Bab El Oued, qui passe pour être celle où les deys envoient puiser l'eau pour leur usage personnel. Le débit des sources est très variable : la source de Sidi Abderrahmane fournit $25 \mathrm{~m}^{3} / \mathrm{j}$, celle du Rempart, dans les fossés de la muraille de Bab Azzoun a un débit de $80 \mathrm{~m}^{3} / \mathrm{j}$, tandis que celle du lavoir de Bab Azzoun, relativement abondante, a un débit de $180 \mathrm{~m}^{3} / \mathrm{j}^{14}$.On constate donc une grande disparité du volume d'eau fourni par ces surgescences.

D'autre part, presque chaque maison possède son puits et obligation est faite aux propriétaires de récupérer à partir des terrasses les eaux pluviales ; elles sont acheminées vers la citerne située dans la majorité des demeures sous le patio, pour éviter d'être coupée de l'eau des aqueducs en cas d'agression, mais aussi de séisme ${ }^{15}$. Ces eaux stockées, sont destinées aux différents usages ménagers, et permettent d'économiser l'eau des sources intramuros, et celle laborieusement collectée par les aqueducs. On ne dénombre pas moins de 2000 citernes et 2 ooo puits pour 3 ooo bâtisses, au début de l'occupation française ${ }^{16}$.

\section{Les aqueducs de la Régence ottomane d'Alger (1518-1620)}

Cette période correspond à celle de l'édification des quatre aqueducs qui alimentent la médina qui en est déjà dotée au XVII ${ }^{\mathrm{e}}$ siècle, époque où son développement urbain atteint son apogée. Ils alimentent en eau la ville jusqu'au début du $\mathrm{XX}^{\mathrm{e}}$ siècle, vers 1910 environ.

Durant toute la Régence ottomane ils sont alimentés par les seules eaux du Sahel proche, utilisées jusque vers 1880, date à laquelle l'apport des eaux de la Mitidja ${ }^{17}$ amorce un tournant décisif dans le ravitaillement en eau d'Alger. Ces aqueducs joueront un rôle important parfois même au-delà de cette date ${ }^{18}$. 


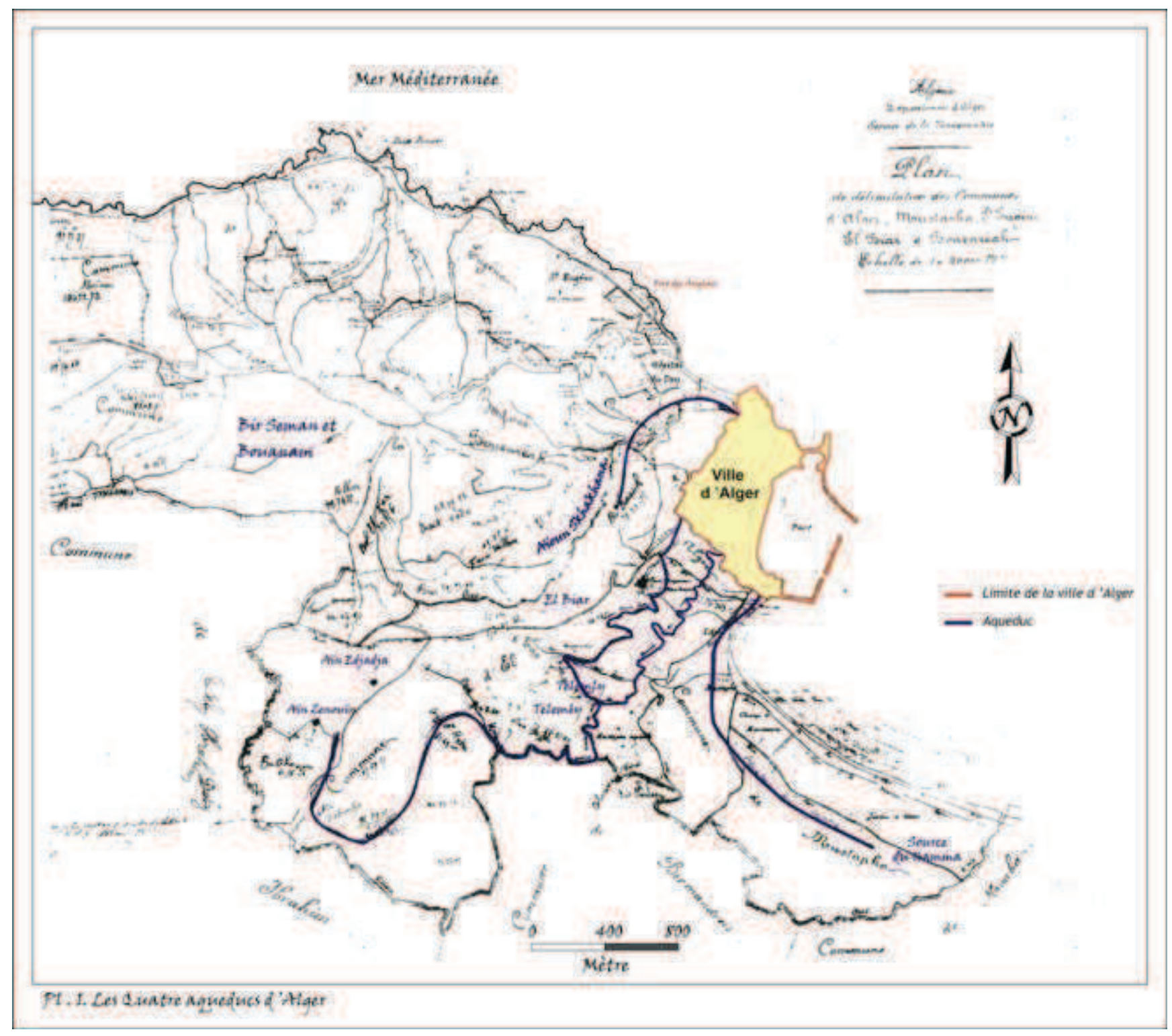

Figure 1. Les quatre aqueducs d'Alger levés en 1874. 


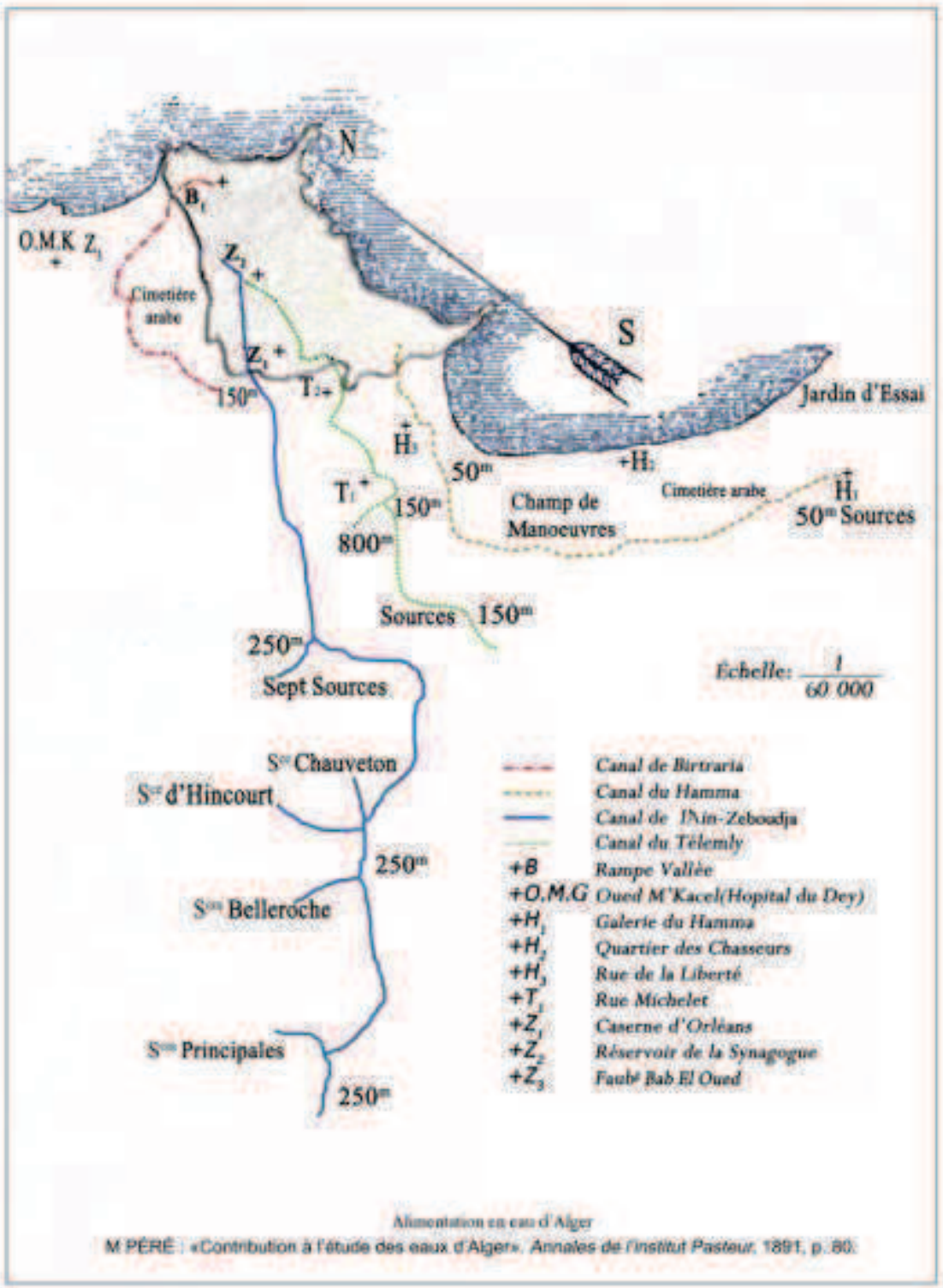

Figure 2. Les quatre grands aqueducs qui alimentent en eau Alger sous la Régence ottomane iusau'au début du XXe siècle 


\section{Originalité technique du souterazi, un régulateur de charge}

Claude-Antoine M. Rozet est le premier auteur à s'intéresser au mode de fonctionnement des aqueducs d'Alger. Il en explique la technique de construction $^{19}$. Ces ouvrages qui traversent les vallées sur un ou plusieurs étages d'arcades, comportent aux extrémités, parfois même dans leur longueur, au milieu de la campagne, un pilier en maçonnerie dans lequel le conduit s'élève verticalement puis redescend le long de ce dernier, formant un souterazi. Il note la ressemblance avec des constructions similaires aux environs de Constantinople et de certaines villes d'Espagne ${ }^{20}$.

Jakob Berggren ${ }^{21}$ observe et décrit les deux techniques de construction des aqueducs des Orientaux; l'une relève de la technique romaine ou grecque du Bas-Empire et l'autre de la technique arabe. À notre sens, il est plus exact d'affirmer que les aqueducs modernes d'Alger empruntent à la fois à la technique des aqueducs sur arcades ${ }^{22}$ et à celle dont relèvent les aqueducs byzantins de Constantinople. Cette technique semble d'ailleurs mieux connue depuis la conquête française de l'Algérie.
Bien antérieurement, comme le précise César Daly, le géographe al-Idrîsî, dans sa description de la

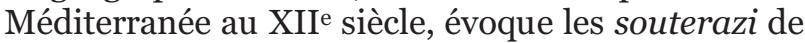
Mérida parmi les vestiges qui témoignent de la puissance et de la grandeur de la colonie romaine Augusta Emérita fondée en 25 avant J.-C :

" Ce qu'il y avait de plus curieux, c'était la manière dont on amenait les eaux [...] par de nombreuses colonnes alignées plus ou moins hautes, selon les exigences du sol, qu'il (Al-Idrisi) nomme ardjâlât ou pieds ${ }^{23}$.

Claude-Antoine M. Rozet observe à tort, que le souterazi ne constitue pas une bonne technique hydraulique :

«Les Algériens s’imaginent accroitre par ce moyen la vitesse de l'eau, ne réfléchissant pas qu'au contraire ils la diminuent en augmentant le développement des tuyaux dans lesquels elle doit circuler $»^{24}$.

Certes d'un point de vue hydraulique, il est illusoire de relever l'eau de quelques mètres pour la faire retomber et couler ensuite plus vite, car l'énergie potentielle gagnée par cette surélévation est perdue par l'énergie cinétique absorbée par la

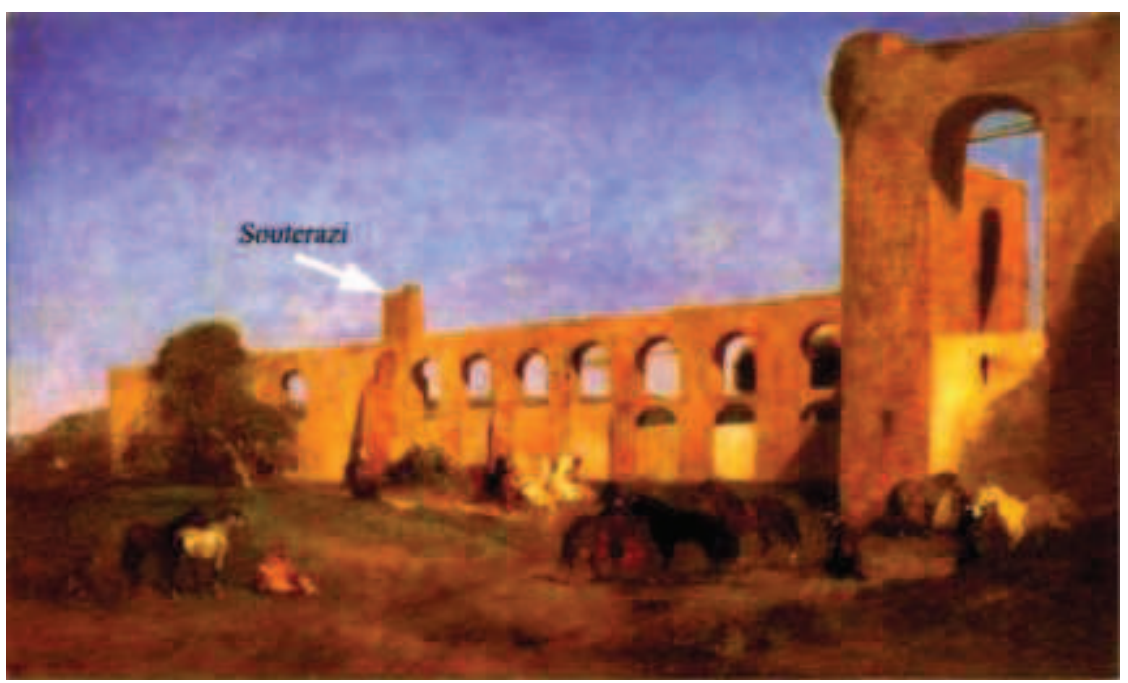

Figure. 3. Représentation d'un souterazi sur une toile du peintre et écrivain français Eugène Fromentin (1820-1876) intitulée "Souvenirs d'Algérie ». Musée des Beaux Arts d'Alger 
paroi du conduit rallongé. Il n'en demeure pas moins que Constantinople et quelques autres villes d'Espagne et du Maghreb ont connu ce type de conduites d'eau.

Cette technique est redécouverte par AntoineFrançois Andréossy, hydrographe, général d'Empire et diplomate français dont l'étude du système des eaux de Constantinople fait référence. Selon cet auteur, soutèrazi ou sou-tèraziçi, est un mot persan et turc composé à partir de sou qui signifie « eau ", et terazi ou balance, soit « équilibre d'eau » 25 ; cet tien, faisait partie des conduites d'eau à siphons renversés. Les tuyaux sont placés dans des rainures masqués par une maçonnerie légère qui les protège mais qui n'empêche pas de les découvrir pour d'éventuelles réparations. Les conduites à souterazi étaient destinées à remplacer les aqueducs à arcades $^{26}$. A.-F. Andréossy décrit les piles qui ont sur leur partie supérieure une ouverture par laquelle l'eau chute brusquement pour laisser échapper l'air entrainé par l'eau, et qui se dégage d'un siphon à l'autre ${ }^{27}$; ces massifs de maçonnerie ou «pyra-

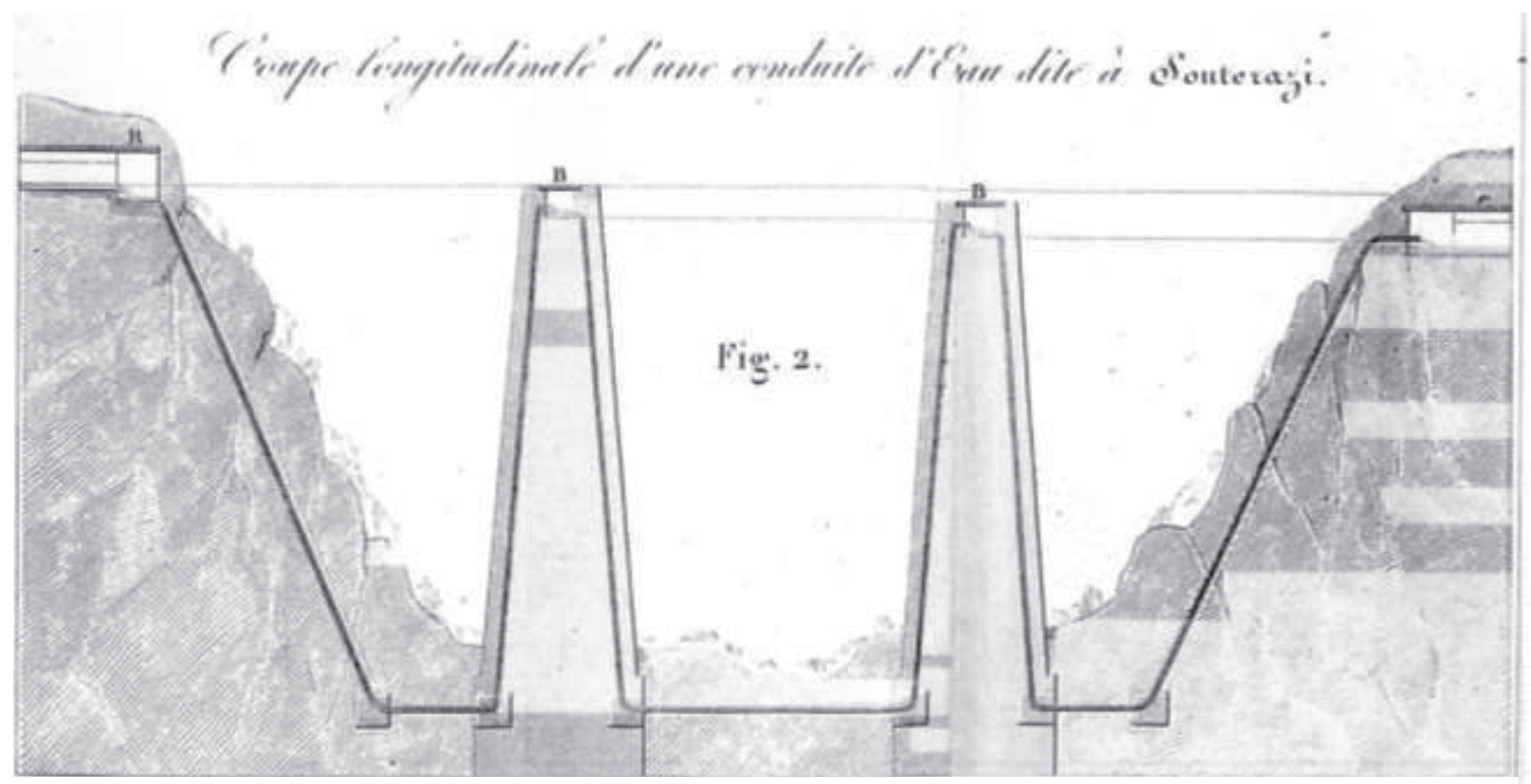

Figure 4 : Coupe longitudinale d'une conduite d'eau à souterazi ; représentation par Raymond Génieys dans son ouvrage Essai sur les moyens de conduire, d'élever et de distribuer les eaux (Paris, chez Carilian-Goery, 1829), planche IV. Il s'agit d'une reprise améliorée de la planche III et IV de l'Atlas d'Antoine-François Andréossy (op. cit. note 25). Ici la conduite est en plomb tandis que celles à souterazi d'Alger et celles du Maghreb d'une manière plus générale, sont en céramique. L'ouverture au sommet permet de chasser l'air dans la conduite et donc de diminuer la pression intérieure. Cette planche est sans échelle mais explique clairement le fonctionnement du système en question. D’après les premiers levés du Génie (premier rapport des Archives nationales de Paris /10/2380 daté du 10 mai 1847), l'aqueduc de l'Aïn Zeboudja présente des souterazi hauts de $8 \mathrm{~m}$.

ouvrage dont la forme évoque un obélisque égyp-

mides hydrauliques » remplacent les aqueducs sur 
arcades dans les terrains accidentés. Leur fonctionnement est conditionné au fait que la source d'eau soit située à un niveau plus élevé de plusieurs pieds $^{28}$ du réservoir de distribution ou du point de distribution d'eau. Cette solution présente l'avantage de résoudre le problème de ventilation nécessaire à l'évacuation de l'air sous pression dans la conduite et de pouvoir être mise en place aussi bien en terrain régulier qu'en terrain très accidenté.

Quant à l'ingénieur Raymond Génieys, dans un traité sur les moyens de conduire l'eau ${ }^{29}$, définit à son tour le souterazi comme un siphon qui permet de faire passer l'eau d'un côté à l'autre d'une vallée, en perdant la charge due à la vitesse de l'eau dans la première partie de l'aqueduc, et celle nécessaire à vaincre les frottements dans les tuyaux. Il s'agit d'une application à grande échelle de la loi des fluides qui ramène constamment l'eau à la même hauteur dans différents tuyaux communicants entre eux; le souterazi remplit réellement l'office de ventouse ${ }^{30}$. Il n'en demeure pas moins qu'il s'agit d'un système ingénieux et économique, relevant de l'art des constructions hydrauliques, encore trop peu connu. Les souterazi suppléent avec avantage aux aqueducs sur arcades: ce procédé permet de réduire la maçonnerie qui peut être de moitié par rapport à celle d'un aqueduc sur arcades, et présente l'intérêt d'être adapté à une adduction en terrain très accidenté, précisément là où la solution du siphon renversé n'est pas réalisable ${ }^{31}$.

Différentes sources écrites et iconographiques relatives à la ville d'Alger permettent une datation approximative de ces ouvrages. Elles témoignent de leur ancienneté et de leur longévité, gage de leur efficacité (voir figure 6).

Nous n'avons pas de renseignement sur les artisans qui travaillent sur ces chantiers hydrauliques, mais une analogie peut être faite avec des travaux similaires de construction de ponts ; Albert Devoulx évoque un acte administratif datant du règne de Hadj 'Ali Pacha, qui relate l'édification du pont du Cheliff, plus grand cours d'eau d'Algérie; ce document donne plusieurs indications sur les ouvriers, les manœuvres, et sur les délais de réalisation: "plus de trois cents musulmans et cent soixante-sept esclaves chrétiens de la nation grecque y furent employés, jour et nuit durant deux mois, et se trouva achevé le 30 redjeb $1229 \mathrm{H} / 18$ juillet $1814 » 3^{2}$. On

sait que sous la Régence, les esclaves publics sont employés principalement à la construction des ponts, aqueducs, remparts, creusement des fossés, quand ils ne sont pas employés comme rameurs de force sur les galères beylières 33 . 
Figure 6 : Les quatre grands aqueducs d'Alger construits à l'époque ottomane. Chronologie approximative établie à partir de différentes sources écrites et iconographiques.

Notes :

a. Rapport Guyot-Duclos, 1840

b. AL-TILIMSANI Muhammad b. Muhammad, AlZahrat al-Nayerat, manuscrit arabe traduit par ROUSSEAU Alphonse, Chroniques de la Régence d'Alger, Alger, Impr. du gouvernement, 1841, p.16 ; voir également NICOLAY (de) Nicolas, Les navigations, pérégrinations et voyages faicts en la Turquie, Anvers, G. Silvius, 1576, p.19. L'auteur qui visite Alger vers 1550 , rapporte que des eaux, près du Fort l'Empereur, "par des conduits souterrains sont menées en cité ».

c. LAUGIER DE TASSY Jacques-Philippe, Histoire du royaume d'Alger, Amsterdam, Henri de Sauzet, 1725, p. 157-158.

d. HAËDO (de) Fray Diego, Topographia e historia general d'Argel, Valladolid, 1612, trad. de l'espagnol par MONNEREAU B. et BERBRUGGER A., Revue africaine, 1871, vol. 15, p. 459-46o.

e. GRAMAYE Jean-Baptiste, trad. Abd el Hadi Ben Mansour, Alger XVI-XVIIe siècle, Journal de JeanBaptiste Gramaye, " évêque d'Afrique », Paris, éd. (s) du Cerf, 1998, p.68.

$f$. Voir pour sa date d'édification l'étude de son inscription épigraphique conservée au Musée des Antiquités d'Alger, figurant dans l'ouvrage de COLIN Gabriel Colin, Corpus des inscriptions arabes et turques de l'Algérie, Paris, Ernest Leroux, t. 1, 1901, inscription 13, datée du $976 \mathrm{H} / 1568-1569$, p.22-23.

g. Ibid., inscription $n^{\circ} 127$ datée de $1217 \mathrm{H} / 1802-1803$, p. $180-181$.

h.GRAMAYE J-B., 1998, op. cit., p.8o ; l'auteur rapporte que ces travaux commandés par Charaf Khûja Pacha sont financés par son prédécesseur Sulayman, grâce à une donation qui équivaut aux deux tiers de son trésor personnel. La contribution au financement des travaux relatifs à la corporation des eaux constitue une ouvre pie par excellence.

i. «Tagarin » désigne les maures originaires de $\mathrm{Va}$ lence, d'Aragon ou de Catalogne. Pour la distinction entre Mudéjares et Tagarins, voir DOZY R., ENGELMANN W. H., Glossaire des mots espagnols et portugais dérivés de l'arabe, 2ème éd., Leyde, E.-J. Brill, $1869, p .321$.

j. DAN Pierre, Histoire de Barbarie et de ses corsaires, Paris, 1649 , p.91.

k. DEVOULX Albert, El Djazair, Histoire d'une cité, Alger, Enag, 2003, p.165, traduction du manuscrit $n^{\circ}$ $1649, f^{\circ} 15$, la BN d'Alger.

\begin{tabular}{|c|c|}
\hline Aqueduc & $\begin{array}{c}\text { Date probable de réalisation / } \\
\text { source de documentation }\end{array}$ \\
\hline Télemly & $\begin{array}{l}\text { - début de réalisation sous Khair Eddine } \\
\text { (règne de 1518-1533) et achevé vers } 1550 \text { sous } \\
\text { le règne de son fils Hasan Pachab. } \\
\text { - Selon Laugier de Tassyc, en 1611, un des } \\
\text { Maures chassés d'Espagneparla Reconquista y } \\
\text { participe par l'acheminement de l'eau d'une } \\
\text { source près du Fort l'Empereur. }\end{array}$ \\
\hline Birtraria & Arab Ahmad vers $1573^{\mathrm{e}}$ \\
\hline $\begin{array}{c}\text { Aïn } \\
\text { Zeboudja }\end{array}$ & $\begin{array}{l}\text { Chanff Khodja vers } 1619 \text { d'après J.-B. } \\
\text { Grammayeh suite à un legs de Sûleyman } \\
\text { Pacha. }\end{array}$ \\
\hline Hamma & $\begin{array}{l}\text { Selon P. Dan, achevé par Mûsa al-Andalusi al- } \\
\text { Himyarite vers } 1611 \text {, sous Kûca Mûstafa Pacha. } \\
\text { Travaux importants sous : } \\
\text { - Ali Pacha en } 1173 \mathrm{H} / 1759 \text {. } \\
\text { - Sid Mohamed Pacha en } 1203 \mathrm{H} / 1788 \text { k. }\end{array}$ \\
\hline
\end{tabular}




\begin{tabular}{|c|c|c|}
\hline $\begin{array}{l}\text { Nombre de } \\
\text { fontaines } \\
\text { desservies en }\end{array}$ & $\begin{array}{c}\text { Zone et principaux édifices alimentés } \\
\text { en eau }\end{array}$ & $\begin{array}{l}\text { Fontaines et établissements } \\
\text { principaux desservis en eau } \\
\text { hors les remparts }\end{array}$ \\
\hline 29 & $\begin{array}{l}\text { La partie médiane de la ville à l'époque ou } \\
\text { Haëdod écrit (1612) : } \\
\text { - } 6 \text { fontaines en ville dont celle du Palais de la } \\
\text { Jenina (résidence des pachas) et la fontaine ou } \\
\text { jet d'eau sur la placette de la Jenina achevée } \\
\text { en } 1580 \text { sous Safer Pacha. } \\
\text { - le réservoir du Bagne du Roi } \\
\text { - la caseme Médée }\end{array}$ & $\begin{array}{l}\text { - les fontaines de Jenan Mûstafa } \\
\text { Pacha (Palais d'Été) } \\
-2 \text { fontaines publiques hors de la } \\
\text { cité. }\end{array}$ \\
\hline 1 & $\begin{array}{l}\text { - uniquement la fontaine publique avec } \\
\text { abreuvoir de la Porte du Ruisseau ou Bab El } \\
\text { Oued } \\
\text { - Dâr an-Nhas. (Fonderie de la } \\
\text { Porte de Bab El Oued) }\end{array}$ & $\begin{array}{l}\text { Les deux forts près de la Porte du } \\
\text { Ruisseau : } \\
\text { - le Fort de Bab El Oued ou } \\
\text { Fort des } 24 \text { heuresf } \\
\text { - le Fort Neuf, plus connu sous } \\
\text { l'appellation de Borj.az-Zúbia. } \\
\text { (Fort des Immondices) de } \\
\text { construction plus tardives. }\end{array}$ \\
\hline 14 & $\begin{array}{l}\text { - la haute ville ainsi que la partie médiane } \\
\text { - } 4 \text { fontaines à la Citadelle ou Palais } \\
\text { du Dey en haut de la ville } \\
\text { - toutes les fontaines le long de la } \\
\text { Rue de la Casbah (Sidi Driss Hamidouche) } \\
\text { - des fontaines au cour de la ville }\end{array}$ & $\begin{array}{l}\text { - la zone des \& Taganns »i } \\
\text { - les Écuries du Dey }\end{array}$ \\
\hline 29 & $\begin{array}{l}\text { La basse ville: } \\
\text { - la caserne Macaron (ancienne } \\
\text { Mosquée Mûqqari'ûn ou Mosquée des Lettrés) } \\
\text { - la Rue des Teinturiers } \\
\text { - le port : avitaillement en eau des } \\
\text { navires }\end{array}$ & $\begin{array}{l}\text { - la Fontaine des Platanes } \\
\text { - les casernes de Mûstafa Pacha } \\
\text { - le Fort Bab Azzoun } \\
\text { - diverses fontaines dans le faubourg } \\
\text { Bab Azzoun } \\
\text { - l'abattoir }\end{array}$ \\
\hline
\end{tabular}




\section{Le regard des colonisateurs}

Pour les premiers ingénieurs chargés de l'alimentation en eau d'Alger, les aqueducs anciens sont obsolètes. La technique des souterazi, relativement moins connue que celle des travaux hydrauliques romains, d'ailleurs nombreux en Algérie, sont largement critiqués par les ingénieurs des Ponts et Chaussées:

«Les aqueducs qui amènent l'eau à Alger sont mal tracés et mal construits ; au lieu d'avoir une pente continuellement descendante de leur source jusqu'à la ville, ils offrent dans plusieurs parties des contre-pentes qui font perdre à l'eau de la vitesse qu'elle a acquise $\gg 34$.

Abondant dans le même sens, Genty de Bussy, premier intendant civil, exprime en ces termes son incompréhension des constructions d'Algérie : "Mais leurs aqueducs, mais la généralité de leurs maisons, tout est à recréer dans un autre système; et en vérité, puisque nous en étions là, peut-être aurait-il mieux valu prendre un grand parti sur-le-champ, et reconstruire successivement toutes les parties de leurs villes $» 35$.

Pourtant ce réseau ne s'avère pas si défaillant, puisqu'il assure l'alimentation en eau de la ville du début de la Régence ottomane jusque vers les années 1880 . Il faudra attendre les premiers rapports des Ponts et Chaussées ${ }^{6}$ pour que soit employé le terme de souterazi.

\section{Conclusion}

La question de l'origine de toute technique, de son attribution chronologique et culturelle est complexe. Celle dont relève les aqueducs d'Alger n'a pas soulevé l'intérêt des chercheurs et archéologues. Ces ouvrages ne sont pas simplement le siège d'un écoulement libre, selon une idée communément admise, mais au contraire, ils présentent des souterazi analogues à ceux de Constantinople et à certains ouvrages d'Espagne: des siphons sont construits sur leur longueur et des chutes d'eau importantes sont réalisées sur leur parcours.

Les quatre grands aqueducs alimentent en eau les abattoirs, les tanneries, les forts et batteries près des portes de la ville et le long de la côte, ainsi que les fontaines publiques. Quant aux aqueducs suburbains, ils desservent les principaux cafés du Fâhs, hauts lieux de la sociabilité masculine 37 , ainsi que les nombreuses fontaines-abreuvoirs sur les routes principales

Tous ces ouvrages hydrauliques relèvent de techniques similaires; parfois des particularités constructives existent ; ponts à double niveau dont certains sont à arcades, et d'autres plus massifs, avec un deuxième niveau plein, tel le pont aqueduc de Tixeraïne, en bon état à ce jour, ou encore celui, aujourd'hui disparu, qui conduit les eaux de l'Aïn Sûliman aux Jardins du Dey, dans le faubourg de Bab El Oued, près de la salpêtrière. Les ponts de ces ouvrages ne représentent certes qu'une petite fraction de la longueur de ces adductions, mais leur étude s'avère précieuse dans la mesure où ils constituent les dernières traces matérielles d'une archéologie hydraulique urbaine mais aussi agricole. Seul peut-être un dernier vestige de souterazi subsiste en l'aqueduc d'irrigation de l'ancien haouch ou domaine Sidi Mohammed al-Kheznadji, structure hydraulique isolée, non dénuée de valeurs paysagère. Visible à partir de l'oued Beni Messous, cet ouvrage dont on ignore le tracé originel et dont la datation reste à établir, mériterait de faire l'objet d'une étude approfondie.

$\mathrm{Au}$ moment de la conquête française le service des eaux dépend, dans un premier temps, du Génie militaire, puis de 1840 à 1846 , du service des Bâtiments Civils qui se contente de remettre en état les conduites d'eau défectueuses, devant rétablir les aqueducs fortement endommagés, au point que l'aqueduc de l'Aïn Zeboudja ne fournit plus d'eau. En 1846, ce service est affecté au corps des Ponts et 
Chaussées ${ }^{38}$ afin d'améliorer le fonctionnement des aqueducs et d'en augmenter les débits. Enfin, en 1880 , les besoins grandissant en eau d'Alger nécessitent d'augmenter le réseau par la collecte des eaux artésiennes 39 de la Mitidja.

${ }^{1}$ RAYMOND André, Grandes villes arabes à l'époque ottomane, Paris, Sindbad, 1985, p. 161.

2 Sous la Régence, le territoire d'Alger est découpé en trois zones : la médina, le Fâhs et les Outans; le « Fâhs » correspond à un territoire inscrit dans un demi-cercle dont Alger est le centre, d'un rayon d'environ 1,5 lieue, soit près de $7 \mathrm{~km}$. Voir TSEF dans l'Algérie en 1841, Paris, Impr. royale, 1842, p.131. Ce territoire, à la veille de la conquête française, est très peu peuplé ; outre les palais d'été et demeures des hauts dignitaires de la Régence, l'on retrouve les représentations des pays étrangers.

3 Le toponyme Birkhadem sera traduit en français par « Puits de la Négresse », tandis que Birmandreis est la contraction de Bir Mûrad Rais ou Puits du capitaine corsaire Mûrad.

4 ROZET Claude-Antoine M., Voyage dans la Régence d'Alger, vol.3, Paris, Arthus Bertrand, 1833, p.95.

5 Voir ANANOU P., "Les populations rurales musulmanes du Sahel d'Alger », Revue africaine, 1953, vol. 97, p. 369, Sahel est un substantif qui, en arabe, est synonyme de rivage, région littorale; le Sahel d'Alger constitue une zone de collines littorales comprise entre les deux limites extrêmes représentées par les bords de l'oued El Harrach à l'est, et l'oued Mazafran avec le mont Chenoua à l'ouest. Il constitue un plateau continu sensiblement horizontal, à une altitude moyenne de $200 \mathrm{~m}$ au-dessus de la mer, et derrière lequel s'étend la plaine de la Mitidja d'une longueur de près de $100 \mathrm{~km}$ et de 5 à $20 \mathrm{~km}$ de largeur.

${ }^{6}$ Pacha est le titre de gouverneur de province dans l'Empire ottoman.

7 FICHEUR Émile, Notice sur la situation géologique et l'origine des eaux d'alimentation de la ville d'Alger, Alger, 1911, p. 17.

8 FICHEUR Émile, Notice de la carte géologique au 1/50.0oo ème, Alger bis, 1904.

9 Ibid.

1o DALLONI Marius, "Le problème de l'alimentation en eau potable de la ville d'Alger », Bulletin de la société de géographie d'Alger et de l'Afrique du Nord, 33ème année, 1er trim.1928, $\mathrm{n}^{\circ} 113$, p.5; au printemps 2008, j'ai pu constater que l'eau de certains puits de la haute Casbah est toujours utilisée pour les tâches ménagères.

${ }^{11}$ VILLE Ludovic, « Notice sur les gîtes minéraux et les matériaux de construction de l'Algérie », Annales des mines, 1869, t.XVI,
Paris, Carilian-Gœury et Victor Dalmont, p.195.

${ }_{12}$ Selon différents auteurs et à la date de leur séjour (entre parenthèse), le nombre des fontaines publiques en ville varie de 100 à 150 : selon DAN Pierre (1634), Histoire de Barbarie et de ses corsaires, Paris, 1649, p. 91, plus de 100 fontaines; selon d'ARVIEUX Laurent (1674-1675), Mémoires du chevalier d'Arvieux, Paris, chez Charles Jean-Baptiste Delespine, vol.5, 1735, p.122,125 fontaines ; selon PLANET Eugène, Correspondance des deys d'Alger avec la cour de France, Paris, F. Alcan, 1889, p.25, 150 fontaines ; selon SHAW Thomas (1738), Voyage dans la Régence d'Alger ou description géographique, physique, philologique de cet état, trad. Mac Carthy, Paris, Marlin, 1830 p.294, 150 fontaines; selon le Rapport du capitaine du Génie Guyot-Duclos, 24 février 1840, Archives SHD, Vincennes, 1H62, 73 fontaines.

${ }^{13}$ CARNET J., L'hiver à Alger au point de vue du traitement des maladies, Paris, V. Masson, 1863, p.55-58. Selon l'auteur, ces données sont fournies par l'ingénieur des Ponts et Chaussées M. de Rougemont, chargé du service de la distribution des eaux d'Alger à cette période.

${ }^{14}$ PASQUALI Eugène, La Casbah d'Alger, ce qu'elle a été, ce qu'elle est, ce qu'elle pourrait être, mémoire multigraphié, Alger, 1951, p. 110

15 GRAMMONT (de) Henri-Delmas, Histoire d'Alger sous la domination turque (1515-1830), Paris, Ernest Leroux, 1887, p.256. L'auteur rapporte que sous les ordres du maréchal $d^{\prime}$ 'Estrées, du $1^{\text {er }}$ au 16 juillet 1686, les galiotes lancent 10420 bombes sur la ville en guise de représailles contre Alger; ces dernières dépassent la ville haute et brisent les aqueducs. Ibid., p.313-314, «[...] la ville manquait d'eau, les derniers tremblements de terre ayant tari les canaux souterrains et les aqueducs. Baba 'Ali (Ali Pacha: 1751-1766), fit rétablir les fontaines et frappa à cet effet d'un nouvel impôt. [...] Les esclaves employés à ces travaux, fort maltraités et privés d'espoir d'être rachetés, par suite de l'énorme prix qu'avaient atteint les rançons, se révoltèrent en masse le 13 janvier 1763 "; voir également Federico Cresti, Description et iconographie de la ville d'Alger au XVIe siècle, ROMM, vol.34, année 1982, p.7. L'auteur rapporte que selon Nicolas de Nicolay, géographe du roi de France, la « grosse tour ronde » ou le Borj Moulay Hasan (le Fort l'Empereur du début de la conquête), est construite afin de protéger la source qui alimente l'aqueduc du Télemly; ceci semble plausible quand on observe le plan d'Alger de la Bibliothèque nationale de France, intitulé Algieri fortificato l'anno 1579 de Henricus Von Schoel. L'on y voit la source ou «fons », en contrebas du fort en question, et l'aqueduc à arcades franchir un ravin pour se rendre en ville. Il est donc antérieur à la date que fournit Laugier de Tassy selon laquelle son édification daterait du début du XVIIe siècle. 
${ }_{16}$ PASQUALI E., 1951, op. cit., p. 110 ; voir par ailleurs, le rapport $\mathrm{n}^{\circ} 1 \mathrm{H} 62, \mathrm{f}^{\circ} 3$, du capitaine du Génie Guyot-Duclos, Archives SHD, Vincennes, Mémoire sur les eaux qui alimentent la ville d'Alger, comptant par contre 1100 citernes d'une capacité de remplissage d'environ $70 \mathrm{~m}^{3}$; ce chiffre est trop général car il correspond à une demeure à patio relativement important, si l'on considère que l'emprise au sol d'une citerne est proportionnelle au tiers de l'emprise au sol de la maison. Or j'ai pu constater, durant les travaux et mesures d'urgence menés au printemps 2008 à la Casbah, que beaucoup de maisons possèdent de petites citernes de 20 à $25 \mathrm{~m}^{3}$, surtout dans la haute ville; par contre les citernes de palais peuvent dépasser la capacité de $300 \mathrm{~m}^{3}$, comme celle du Palais du Dey; celle de Dâr Khedaouedj al-'Amia, actuel Musée des arts traditionnels et populaires est de près de $150 \mathrm{~m}^{3}$.

17 DALLONI M., 2008, op. cit., note13, p. 10 -11; voir CRESTI Federico, Le système de l'eau à Alger pendant la période ottomane (XVIe-XIXe siècles). Environnemental Design, 1-2, Rome, 1992, p. 42-53; voir également René Lespes, Alger, étude de géographie et d'histoire urbaine, Paris, F. Alcan, 1930, p.446.

${ }^{18}$ La première zone industrielle se situe au quartier du Ruisseau, à l'est d'Alger, le long de la voie ferrée et de la route moutonnière, ancienne route de Constantine. Vers 1920, les eaux du Hamma sont affectées à l'alimentation de la brasserie d'Alger, dans le quartier Belouizdad, ex Belcourt, zone du Mustapha inférieur desservie en eau par le même aqueduc; voir les articles du 5 et 20 juillet 1920 du Bulletin Municipal de la ville d'Alger.

19 ROZET C-A. M., 1833, op. cit., p.95.

${ }^{20} \mathrm{Ibid}$.

${ }^{21}$ BERGGREN Jakob, Guide français-arabe vulgaire des voyageurs et des Francs en Syrie et en Egypte, Upsal, chez Leffler et Sebell, 1844, p.56-57. L'auteur qualifie ce système de technique des aqueducs arabes reposant entièrement sur « la théorie des tuyaux », tout en fournissant de plus amples détails relatifs à la réalisation de ces derniers.

22 Voir également la même conclusion à propos des aqueducs à souterazi et à arcades, dans DALY César, Revue générale de l'architecture, des ingénieurs, des archéologues, des industriels et des propriétaires, vol.1, Paris, Paulin et Hetzel, 1840, p.127.

${ }_{23}$ Ibid.

24 ROZET C-A. M., 1833, op. cit., p.95.

25 ANDRÉOSSY Antoine-François, Constantinople et le Bosphore de Thrace, pendant les années1812, 1813 et1814 et pendant l'année 1826, Paris, Théophile Barrois et Benjamin Duprat, 1828, p.435.

${ }^{26}$ Ibid., p.520.

${ }_{27}$ Ibid., p.463.

${ }^{28}$ Un pied $\sim 0,30 \mathrm{~m}$
29 GÉNIEYS Raymond, Essai sur les moyens de conduire, d'élever et de distribuer les eaux, Paris, Carilian-Gœury, 1829, p.51et planche IV.

3o Ibid.

${ }^{31}$ « The aqueducts and water-works of Constantinople», from the french of count Andreossy, dans The parterre of fiction, poetry, history, and general literature, vol.III, London, Effingham Wilson, junior, $\mathrm{n}^{\mathrm{0}} 76,1835$, London, p.378. L'auteur mentionne l'existence de simples souterazi et de souterazi plus complexes (Compoud souterazi).

${ }^{32}$ DEVOULX Albert, Tachrifat, recueil de notes historiques sur l'administration de l'ancienne Régence d'Alger, Alger, Impr. du gouvernement, 1852, p.79.

33 FONTENAY Michel, « Routes et modalités du commerce des esclaves dans la Méditerranée des temps modernes (XVIe, XVIIe et XVIIIe siècles) ", Revue historique, 2006/ 4, n640, PUF, p.814.

34 Tableau de la situation des établissements français dans l'Algérie en 1838, Paris, Impr. royale, 1839, p.16.

35 GENTY DE BUSSY M.- P., De l'établissement des Français dans la Régence d'Alger et des moyens d'en assurer la prospérité, vol.1, Paris, Impr. Firmin Didot frères, 1839, p.296.

${ }^{36}$ Le premier rapport des Archives nationales de Paris /10/2380 daté du 10 mai 1847.

37 CARLIER Omar, "Le café maure, sociabilité masculine et effervescence citoyenne, (Algérie XVIIIe-XXe siècles)», dans Annales, Économies, Sociétés, Civilisation, 1990-4, p.975.

38 Tableau de la situation des établissements français dans l'Algérie, 1846-1847-1848-1849, Paris, Impr. nationale, 1851, p.347.

39 Les eaux des puits artésiens jaillissent spontanément sous la mise en pression de l'aquifère. Ce type de puits artésiens existe bien avant le puits d'Artois et la corporation des puisatiers ou plongeurs (ghettas) et leurs prouesses sont légendaires à cause de la dangerosité du métier. Voir FOURNEL Henri, Richesses minérales de l'Algérie, Impr. nationale, 1850, t. 2, Paris, Carilian-Gœury et V. Dalmont, p. X. Cet ingénieur des Mines signale leur existence dans les oasis de l'Oued Righ, de Temacin et d'Ouargla. 